\title{
The 2012 Core Content of Medical Toxicology
}

\author{
Lewis S. Nelson • Beth A. Baker • Kevin C. Osterhoudt • \\ Curtis P. Snook - The Medical Toxicology Core Content \\ Task Force for the Medical Toxicology Subboard • \\ Julia N. Keehbauch • for the American Board of \\ Emergency Medicine
}

Published online: 30 May 2012

(C) This article is being published without copyright 2012

Keywords Core content $\cdot$ Medical toxicology $\cdot$ Curriculum

\section{Preamble}

In December 2011, the Medical Toxicology Subboard, composed of representatives from emergency medicine, pediatrics, and preventive medicine, approved a revised Core Content of

Medical Toxicology Subboard Members: Frederick Fung, M.D., Daniel A. Goldstein, M.D., Rama B. Rao, M.D., Anne-Michelle Ruha, M.D., and Saralyn R. Williams, M.D.

James H. Jones, M.D. (American Board of Emergency Medicine Board Liaison), Julia N. Keehbauch (American Board of Emergency Medicine)

The Core Content of Medical Toxicology is used with the permission of the American Board of Emergency Medicine, American Board of Pediatrics, and American Board of Preventive Medicine, copyright 2012.

\section{S. Nelson}

New York University School of Medicine,

New York, NY, USA

\section{B. A. Baker}

University of Minnesota School of Public Health,

Minneapolis, MN, USA

\section{K. C. Osterhoudt}

The Children's Hospital of Philadelphia,

Philadelphia, PA, USA

C. P. Snook

Cincinnati Veterans Administration Medical Center,

Cincinnati, Ohio, USA

\section{J. N. Keehbauch $(\bowtie)$}

American Board of Emergency Medicine,

3000 Coolidge Road,

East Lansing, MI 48823, USA

e-mail: jkeehbauch@abem.org
Medical Toxicology. The Core Content encompasses the specialty of medical toxicology and outlines the areas of knowledge considered essential for the practice of medical toxicology. Functionally, the Core Content provides the organizational framework for the development of the medical toxicology certification and cognitive expertise examinations, and details the knowledge to be tested on those examinations, beginning with the 2014 examinations. In addition, the Core Content may serve as a template for the development of medical toxicology fellowship curricula. The previous version, initiated in 2000, approved in 2002, and published in 2004, ${ }^{1}$ will be retired and replaced by this new version.

The first Medical Toxicology Core Content was developed to assist in the construction of the first examination in 1994. This document consisted of 22 major content areas and was organized, in part, by toxicant classification. Listed under most of these major content areas were exhaustive lists of drugs and toxicants of which a medical toxicologist was expected to have knowledge.

In 2000, the Medical Toxicology Subboard embarked on a major revision of the original Core Content. This undertaking was initiated to update the 1994 Core Content, as well as to improve the framework that conceptualizes the expanding breadth of medical toxicology. In addition, the Subboard wanted to devise a conceptual document that would accommodate future discoveries and changes. A task force of Medical Toxicology Subboard members was convened to develop and draft the revised Core Content, and comments were solicited from stakeholders.

\footnotetext{
${ }^{1}$ Wax PM, Ford MD, Bond GR, Kilbourne EM, Walter FG; for Medical Toxicology Core Content Task Force; Avery AN, Clark RF, Liebelt EL; for Medical Toxicology Subboard Members. The core content of medical toxicology. Ann Emerg Med. 2004;43:209-214.
} 
The latest Core Content Task Force was established in 2009 to propose updates to the 2004 Core Content. Development included both extensive discussion by the Medical Toxicology Subboard and the use of a survey of practitioners, which will be reported separately. During the process, input was solicited from the American College of Medical Toxicology, American Academy of Clinical Toxicology, and other interested stakeholders, such as diplomates, medical toxicology fellowship directors, and related organizations.

The Core Content now contains six distinct subject areas: (1) principles of toxicology, (2) toxins and toxicants, (3) clinical assessment, (4) therapeutics, (5) assessment and population health, and (6) analytical and forensic toxicology. The most significant change was the addition of an entire subject area, clinical assessment. Since many clinical encounters begin with a symptom or clinical finding rather than with the knowledge of a specific toxin, the inclusion of this section better aligns the Core Content with clinical practice.

As with previous revisions of the Core Content, the Medical Toxicology Core Content Task Force grappled with what level of detail to include in the document. In this latest revision, the Core Content Task Force revised many of the sections of the previous version, both adding and reducing detail as appropriate. The rationale for adjusting the level of detail provided was the perception that some areas, such as the occupational toxicants, were too specific and needed to be broadened while others, such as drugs of abuse, were not sufficiently inclusive. Given the ongoing introduction of new pharmaceuticals and chemicals, yearly updating of the document to include a definitive list of all toxins and toxicants becomes increasingly unwieldy. A few representative examples are included in many of the drug and toxicant classes for clarity, but the scope of medical toxicology and the examination is not limited to these examples. The Subboard believes it is reasonable that test items can be developed on any agent that could be classified either under a broader category (e.g., drugs that affect the cardiovascular system) or a narrower category (e.g., antihypertensives).

The Core Content is intended to be a living document, in keeping with the ever-evolving practice of medical toxicology. The Medical Toxicology Subboard anticipates regular updates to the Core Content. In order to allow the update process to accurately reflect this evolution, the Subboard welcomes input from its stakeholders. Suggested changes to the Core Content can be addressed to examcontent@abem.org.

\section{Medical Toxicology \\ Core Content \\ 2012}

Medical Toxicology Major Core Content Categories

1.0 Principles of Toxicology

2.0 Toxins and Toxicants

2.1 Drugs

2.2 Drugs of Abuse

2.3 Industrial, Household, and Environmental Toxicants

2.4 Natural Products

2.5 Warfare and Terrorism

2.6 Radiological

3.0 Clinical Assessment

4.0 Therapeutics

5.0 Assessment and Population Health

6.0 Analytical and Forensic Toxicology

\section{Medical Toxicology Core Content}

\section{Part 1: Principles of Toxicology}

1.1 Pharmacology/Toxicology

\subsubsection{Pharmacokinetics/Toxicokinetics}

1.1.1.1 Bioavailability and Absorption

1.1.1.2 Clearance

1.1.1.3 Distribution

1.1.1.4 Elimination

1.1.1.5 Metabolism

1.1.1.6 Models (e.g., Compartmental, Physiologic)

1.1.2 Pharmacodynamics/Toxicodynamics

1.1.2.1 Dose/Concentration Relationship to Effect

1.1.2.2 Receptor Agonism/Antagonism

1.1.2.3 Receptor Regulation

1.1.2.4 Structure-Activity Relationship

\subsubsection{Adverse Effects}

1.1.3.1 Idiosyncratic

1.1.3.2 Mechanistic (Predictable)

1.1.4 Interactions

1.1.4.1 Xenobiotic-Environment

1.1.4.2 Xenobiotic-Food

1.1.4.3 Xenobiotic-Xenobiotic (e.g., Drug-Drug)

1.1.5 Proteomics

1.1.6 Tolerance and Withdrawal 
1.1.6.1 Behavioral (Pharmacodynamic) Tolerance

1.1.6.2 Biologic (Pharmacokinetic) Tolerance

1.1.6.3 Dependence

1.1.7 Immunologic Response (e.g., Antibodies, Cytokines)

1.1.8 Pharmacogenetics/Toxicogenetics

1.1.8.1 Assessment and Profiling of Polymorphisms

1.1.8.2 Role in Adverse Drug Events

1.2 Molecular Components/Mechanisms

1.2.1 Channels and Pumps

1.2.2 Enzymes and Transport Proteins (e.g., Methemoglobin, G6PD)

1.2.3 Glycolysis and Oxidative Phosphorylation

1.2.4 Membranes

1.2.5 Neurotransmitters

1.2.5.1 Receptor Isoforms and Subtypes

1.2.5.2 Regulation and Messengers

1.2.5.3 Signal Transduction

1.2.6 Other Metabolic Pathways (e.g., Amino Acids and Urea Cycle)

1.3 Cytotoxic Mechanisms (e.g., Apoptosis, Microtubular Dysfunction)

1.4 Principles of Radiation (e.g., Decay, Units)

1.5 Mutagenesis and Carcinogenesis

1.5.1 Mutagenesis

1.5.1.1 Chromosome Aberrations (Structural, Numerical)

1.5.1.2 Gene Mutation (Oncogenes, Tumor Suppressor Genes)

1.5.2 Development of Neoplasia

1.5.2.1 Initiation (e.g., Genotoxic Mechanisms)

1.5.2.2 Procarcinogens and Conversion to Carcinogens (e.g., Biotransformation)

1.5.2.3 Progression (e.g., Growth, Invasiveness)

1.5.2.4 Promotion (e.g., Nongenotoxic Mechanisms)

1.5.3 Inhibition of Carcinogenesis Modulating Factors

1.5.3.1 Endogenous Factors (e.g., Age, Sex, Immune Status, Hormones)

1.5.3.2 Exogenous Factors (e.g., Diet, Radiation)

1.5.4 Interactive Carcinogenesis

1.5.4.1 Xenobiotic-Gene Interactions

1.5.4.2 Xenobiotic-Physical Interactions

1.5.4.3 Xenobiotic-Radiation Interactions

1.5.4.4 Xenobiotic-Viral Interactions

1.6 Mechanisms of Reproductive and Developmental Toxicity

1.6.1 Conception Impairment, Mutagenesis, and Teratogenesis

1.6.1.1 Chromosomal and Gene Abnormalities
1.6.1.2 Effects on Gametogenesis and Gametes

1.6.1.3 Effects on Gonads

1.6.1.4 Spontaneous Abortion and Perinatal Death

1.6.2 Factors Determining Fetal or Infant Exposure to Agents

1.6.2.1 Breast Milk Transfer

1.6.2.2 Placental Transfer

1.6.2.3 Xenobiotic Disposition (e.g., Maternal Xenobiotic Disposition, Fetal Pharmaco- kinetics)

1.6.3 Offspring Effects

1.6.3.1 Cancer

1.6.3.2 Congenital Anomalies and Malformations

1.6.3.3 Development of Infant/Child

1.6.3.4 Genetic Mutations

1.6.4 Pharmacology of the Neonate

1.6.5 Pharmacology of Pregnancy

\section{Part 2: Toxins and Toxicants}

2.1 Drugs

2.1.1 Analgesics, Anti-Inflammatory Drugs

2.1.1.1 Acetaminophen

2.1.1.2 NSAIDs

2.1.1.3 Opioids

2.1.1.4 Salicylates

2.1.1.5 Others (e.g., Antigout Drugs, Gold)

2.1.2 Antimicrobials

2.1.2.1 Antibiotics

2.1.2.2 Antifungals

2.1.2.3 Antimycobacterials

2.1.2.4 Antiparasitics

2.1.2.5 Antiprotozoals

2.1.2.6 Antiretrovirals

2.1.2.7 Antiseptics

2.1.2.8 Antivirals

2.1.3 Chemotherapeutic Drugs

2.1.3.1 Alkylators

2.1.3.2 Antimetabolites

2.1.3.3 Hormones

2.1.3.4 Natural Drugs (e.g., Vinca Alkaloids, Antibiotics)

2.1.3.5 Miscellaneous (e.g., Platinum, Hydroxyurea)

2.1.4 Diagnostic Aids (e.g., Contrast Agents)

2.1.5 Drugs that Affect Cholesterol and Lipids

2.1.6 Drugs that Affect the Cardiovascular System

2.1.6.1 Antidysrhythmics

2.1.6.1.1 Calcium Channel Blockers

2.1.6.1.2 Cardioactive Steroids

2.1.6.1.3 Potassium Channel Blockers

2.1.6.1.4 Sodium Channel Blockers

2.1.6.2 Antihypertensives 
2.1.6.2.1 Angiotensin System Modulators

2.1.6.2.2 Beta Adrenergic (and Mixed $\alpha, \beta$ ) Antagonists

2.1.6.2.3 Centrally Acting Alpha Receptor Agonists

2.1.6.2.4 Diuretics

2.1.6.2.5 Vasodilators (e.g., Nitrates, Nitrites)

2.1.6.3 Inotropes

2.1.7 Drugs that Affect the Respiratory System

2.1.7.1 Bronchodilators and Respiratory Stimulants (e.g., Methylxanthines)

2.1.7.2 Antitussives and Cold Preparations

2.1.8 Drugs that Affect the Endocrine System

2.1.8.1 Antidiabetic Drugs

2.1.8.1.1 Insulin

2.1.8.1.2 Oral Hypoglycemics

2.1.8.1.3 Others (e.g., Metformin, Glitazones)

2.1.8.2 Bone Active Drugs (e.g., Calcitonin, Bisphosphonates)

2.1.8.3 Electrolytes and Minerals

2.1.8.4 Glucocorticoids

2.1.8.5 Sex Hormones, Growth Hormones, and Anabolic Steroids

2.1.8.6 Thyroid Drugs

2.1.8.7 Vasopressin And Somatostatin Analogues

2.1.9 Drugs that Affect the Gastrointestinal System

2.1.9.1 Antidiarrheals

2.1.9.2 Antiemetics

2.1.9.3 Drugs Used for Biliary and Pancreatic Diseases (e.g., Bile Salts, Pancreatic Enzymes)

2.1.9.4 Drugs Used for Inflammatory Bowel Disease

2.1.9.5 Drugs Used to Treat Acid-Peptic Diseases

2.1.9.6 Laxatives

2.1.9.7 Promotilics

2.1.10 Drugs that Affect the Hematologic System

2.1.10.1 Anticoagulants

2.1.10.2 Antifibrinolytics

2.1.10.3 Antiplatelet Drugs

2.1.10.4 Blood Stimulating Drugs (e.g., Erythropoietin)

2.1.10.5 Drugs Used to Treat Bleeding (e.g., Clotting Factors, Antiplasmin Drugs)

2.1.10.6 Iron

2.1.10.7 Thrombolytics

2.1.11 Drugs that Affect the Immune System (e.g., Interferon, Cyclophosphamide)

2.1.12 Drugs that Affect the Nervous System

2.1.12.1 Anesthetics

2.1.12.1.1 Inhalational and Sedative Anesthetics (e.g., Nitrous Oxide, Propofol)
2.1.12.1.2 Local Anesthetics

2.1.12.2 Anticonvulsants

2.1.12.3 Antiparkinsonism Drugs

2.1.12.4 Drugs that Affect Autonomic Homeostasis

2.1.12.4.1 Anticholinergics

2.1.12.4.2 Antihistamines

2.1.12.4.3 Antiserotonergics

2.1.12.4.4 Cholinergics (e.g., Nicotine)

2.1.12.4.5 Ergot and Derivatives

2.1.12.4.6 Serotonin Agonists and Other Proserotonergics (e.g., Dextromethorphan)

2.1.12.5 Ethanol

2.1.12.6 Muscle Relaxants

2.1.12.7 Neuromuscular Blockers

2.1.12.8 Psychoactive Drugs and Hallucinogens (e.g., Marijuana, LSD)

2.1.12.9 Psychotropics

2.1.12.9.1 Antidepressants

2.1.12.9.2 Antipsychotics

2.1.12.9.3 Anxiolytics and SedativeHypnotics

2.1.12.9.4 Mood Stabilizers

2.1.13 Pharmaceutical Additives (e.g., Excipients)

2.1.14 Veterinary Products

2.1.15 Vitamins

2.2 Drugs of Abuse

2.2.1 CNS Depressants (e.g., GHB, SedativeHypnotics)

2.2.2 Dissociatives

2.2.3 Hallucinogens

2.2.4 Inhalants (e.g., Hydrocarbons)

2.2.5 Opioids

2.2.6 Sympathomimetics (e.g., Amphetamines, Cocaine)

2.3 Industrial, Household, and Environmental Toxicants

2.3.1 Airborne Solids

2.3.1.1 Asbestos

2.3.1.2 Coal Dust

2.3.1.3 Organic Dust

2.3.1.4 Silica

2.3.1.5 Other Minerals (e.g., Man-Made Mineral Fibers)

2.3.2 Cleansers and Caustics

2.3.2.1 Acids

2.3.2.2 Alkali

2.3.2.3 Bleach

2.3.2.4 Detergents and Soaps

2.3.2.5 Disinfectants and Topical Anti-Infectives

2.3.2.6 Swimming Pool Products

2.3.3 Food Additives (e.g., MSG, Sulfites)

2.3.4 Household Products 


\subsubsection{Aquarium Products}

2.3.4.2 Art Products

2.3.4.3 Batteries

2.3.4.4 Cosmetics

2.3.4.5 Dental Products

2.3.4.6 Hair Products

2.3.4.7 Personal Hygiene Products

2.3.5 Hydrocarbons/Solvents/Fuels

2.3.5.1 Aldehydes

2.3.5.2 Alcohols and Glycols

2.3.5.2.1 Diethylene Glycol

2.3.5.2.2 Ethylene Glycol

2.3.5.2.3 Glycol Ethers

2.3.5.2.4 Isopropanol

2.3.5.2.5 Methanol

2.3.5.3 Aliphatic Hydrocarbons

2.3.5.3.1 Hexane and Congeners

2.3.5.3.2 Mixtures (e.g., Gasoline, Kerosene)

2.3.5.4 Aromatic Hydrocarbons
2.3.5.4.1 Benzene
2.3.5.4.2 Polycyclic Aromatic Hydrocarbons
2.3.5.4.3 Toluene

2.3.5.5 Halogenated Hydrocarbons

2.3.5.5.1 Carbon Tetrachloride

2.3.5.5.2 Chloroform

2.3.5.5.3 Methylene Chloride

2.3.5.5.4 Perchloroethylene

2.3.5.5.5 Trichloroethylene

2.3.5.5.6 Vinyl Chloride

2.3.5.6 Hydrazines

2.3.5.7 Ketones

2.3.5.8 Peroxides

2.3.5.9 Terpenes

2.3.6 Metals/Metalloids

2.3.6.1 Arsenic (Including Arsine)

2.3.6.2 Barium

2.3.6.3 Beryllium

2.3.6.4 Cadmium

2.3.6.5 Chromium

2.3.6.6 Cobalt

2.3.6.7 Copper

2.3.6.8 Lead

2.3.6.9 Manganese

2.3.6.10 Mercury

2.3.6.11 Nickel

2.3.6.12 Thallium

2.3.6.13 Other Metals/Metalloids

2.3.7 Pesticides

2.3.7.1 Fumigants and Sterilants

2.3.7.2 Fungicides

2.3.7.3 Herbicides

2.3.7.4 Insecticides and Repellents

2.3.7.4.1 Carbamates

\subsection{DEET}

2.3.7.4.3 Moth Repellants

2.3.7.4.4 Organochlorines

2.3.7.4.5 Organophosphates

2.3.7.4.6 Pyrethrins and Pyrethroids

2.3.7.5 Rodenticides

2.3.7.5.1 Anticoagulant

2.3.7.5.2 Non-anticoagulant

2.3.7.6 Other (e.g., Molluscides)

2.3.8 Pollutants

2.3.8.1 Air Pollutants (e.g., Respirable Particulates, Ozone)

2.3.8.2 Persistent Organic Pollutants (e.g., Polychlorinated Biphenyls, Dibenzodioxins)

2.3.8.3 Water Pollutants (e.g., Trihalomethanes)

2.3.9 Toxic Gases

2.3.9.1 Cellular Asphyxiant Gases

2.3.9.1.1 Carbon Monoxide

2.3.9.1.2 Cyanide

2.3.9.1.3 Hydrogen Sulfide

2.3.9.2 Irritant Gases

2.3.9.2.1 Chlorine

2.3.9.2.2 Nitrogen Oxides

2.3.9.2.3 Ozone

2.3.9.2.4 Phosgene

2.3.9.2.5 Sulfur Oxides

2.3.9.3 Simple Asphyxiants (e.g., Methane, Nitrogen)

2.3.10 Miscellaneous Toxicants

2.3.10.1 Acrolein

2.3.10.2 Acrylates and Acrylamides

2.3.10.3 Amines, Diamines, and Amides

2.3.10.4 Aniline Compounds

2.3.10.5 Azides

2.3.10.6 Bromide Compounds

2.3.10.7 Butadienes

2.3.10.8 Carbon Disulfide

2.3.10.9 Chlorates

2.3.10.10 Coal Tar Products

2.3.10.11 Epichlorohydrin

2.3.10.12 Ethylene Dibromide (EDB)

2.3.10.13 Fluorides and Fluorocarbons

2.3.10.14 Fuels

2.3.10.15 Isocyanates (e.g., Toluene Diisocyanate)

2.3.10.16 Maleic Anhydride (MDA)

2.3.10.17 Mercaptans

2.3.10.18 Nanoparticulates/Nanotoxicology

2.3.10.19 Nitriles

2.3.10.20 Phosphorus/Phosphides

2.3.10.21 Phthalates

2.3.10.22 Polymers and Resins

2.3.10.23 Styrene

2.3.10.24 Trimellitic Anhydride

2.3.10.25 Other Miscellaneous Toxicants 
2.4 Natural Products

2.4.1 Dietary Supplements

2.4.1.1 Herbal Products

2.4.1.2 Performance Enhancing Substances (e.g., Anabolics, Creatine)

2.4.1.3 Vitamins and Minerals

2.4.2 Food Poisoning

2.4.2.1 Bacteria and Bacterial Toxins

2.4.2.2 Marine

2.4.3 Fungi

2.4.3.1 Mushrooms

2.4.3.2 Other Fungal Toxins (e.g., Aflatoxins, Trichothecene Mycotoxins)

2.4.4 Plants

2.4.4.1 Cardiovascular Toxic Plants (e.g., Aconitum napellus, Nerium oleander)

2.4.4.2 Cutaneous/Mucus Membrane Toxic Plants (e.g., Toxicodendron spp., Dieffenbachia spp.)

2.4.4.3 Gastrointestinal Toxic Plants (e.g., Phytolacca americana, Solanum spp.)

2.4.4.4 Hepatotoxic Plants (e.g., Blighia sapida, Symphytum spp.)

2.4.4.5 Multi-system Toxic Plants (e.g., Prunus spp., Ricinus communis)

2.4.4.6 Neurotoxic Plants (e.g., Datura spp., Nicotiana tabacum)

2.4.5 Toxic Envenomations

2.4.5.1 Arthropods

2.4.5.1.1 Hymenoptera

2.4.5.1.2 Scorpions

2.4.5.1.3 Spiders

2.4.5.2 Marine Animals

2.4.5.3 Reptiles

2.4.5.3.1 Elapidae

2.4.5.3.2 Viperidae

2.4.5.3.3 Other Snakes

2.4.5.3.4 Venomous Lizards

2.4.5.4 Amphibians (e.g., Toads, Frogs)

2.5 Warfare and Terrorism

2.5.1 Biological

2.5.1.1 Bacteria (e.g., Anthrax, Plague)

2.5.1.2 Toxins (e.g., Botulinum, Staph B)

2.5.1.3 Viruses (e.g., Smallpox)

2.5.2 Chemical

2.5.2.1 Acetylcholinesterase Inhibitors (e.g., Sarin, Soman, VX)

2.5.2.2 Blister Agents (e.g., Mustard)

2.5.2.3 Incapacitating Agents (e.g., Calmatives, BZ [3-Quinuclidinyl Benzilate])

2.5.2.4 Tear Gases (e.g., Pepper Spray)
2.6 Radiological

2.6.1 Radiation Sources (e.g., Industrial, Medical)

2.6.2 Specific Radionuclides (e.g., Cesium, Iodine, Polonium, Radon)

\section{Part 3: Clinical Assessment}

3.1 Toxicologic Syndromes (e.g., Cholinergic, Opioid)

3.2 Vital Sign Abnormalities/Syndromes

3.2.1 Blood Pressure

3.2.2 Heart Rate and Rhythm

3.2.3 Temperature

3.2.4 Ventilation and Oxygenation

3.3 Differential Diagnosis by Clinical Finding

3.3.1 Cardiac

3.3.1.1 Cardiovascular Collapse

3.3.1.2 Chest Pain

3.3.1.3 Palpitation

3.3.1.4 Syncope

3.3.2 Dermatologic

3.3.2.1 Color Change (e.g., Cyanosis, Jaundice)

3.3.2.2 Hair and Nail Abnormalities

3.3.2.3 Rash and Other Cutaneous Reactions

3.3.3 Gastrointestinal

3.3.3.1 Abdominal Pain

3.3.3.2 Diarrhea/Constipation

3.3.3.3 GI Bleed

3.3.3.4 Hepatic

3.3.3.4.1 Fulminant Hepatic Failure

3.3.3.4.2 Hepatic Encephalopathy

3.3.3.5 Pancreatitis

3.3.3.6 Vomiting

3.3.4 Hematologic

3.3.4.1 Red Blood Cell Abnormalities (e.g., Anemia, Hemolysis)

3.3.4.2 White Blood Cell Abnormalities (e.g., Leukocytosis, Leukopenia)

3.3.4.3 Hemostatic Disorders (e.g., Coagulopathy, Thrombocytopenia)

3.3.5 Musculoskeletal

3.3.5.1 Rhabdomyolysis

3.3.5.2 Skeletal Abnormalities

3.3.6 Neurologic

3.3.6.1 Ataxia

3.3.6.2 Chronic Pain Syndromes

3.3.6.3 Delirium

3.3.6.4 Depressed Mental Status/Coma

3.3.6.5 Headache

3.3.6.6 Movement Disorder

3.3.6.7 Neuropathy/Paresthesia

3.3.6.8 Rigidity

3.3.6.9 Seizure 


\subsubsection{Tremor}

3.3.6.11 Weakness/Paralysis

3.3.6.12 Withdrawal Syndromes (e.g., Alcohol, Opioid)

3.3.7 Psychiatric

3.3.7.1 Agitation

3.3.7.2 Depression

3.3.7.3 Hallucinations

3.3.7.4 Psychosis

3.3.8 Pulmonary

3.3.8.1 Cough

3.3.8.2 Lung and Related Cancer

3.3.8.3 Pulmonary Edema/Acute Lung Injury

3.3.8.4 Restrictive Lung Disease

3.3.8.5 Smoke Inhalation

3.3.8.6 Wheezing/Bronchospasm

3.3.9 Renal/Genitourinary

3.3.9.1 Acute Renal Failure (e.g., Acute Tubular Necrosis)

3.3.9.2 Fertility Abnormalities

3.3.9.3 Nephritis/Nephrosis

3.3.9.4 Obstruction/Renal stone

3.3.9.5 Sexual Dysfunction

3.3.10 Special Senses

3.3.10.1 Vision Loss/Changes

3.3.10.2 Pupil Changes

3.3.10.3 Hearing Loss/Tinnitus

3.4 Pediatric and Reproductive Syndromes

3.4.1 Child Abuse (e.g., Munchausen by Proxy)

3.4.2 Developmental Disorders (e.g., Autism, ADHD)

3.4.3 Teratogenesis (e.g., Fetal Alcohol Syndrome)

3.5 Radiation Syndromes

3.5.1 Acute Radiation Exposure

3.5.1.1 Local

3.5.1.2 Systemic

3.5.2 Carcinogenesis/Mutagenesis

3.6 Syndromes Attributed to the Environment, Not Specified Elsewhere

3.6.1 Event-specific Syndromes (e.g., Gulf War Syndrome, World Trade Center cough)

3.6.2 Mold

3.6.3 Multiple Chemical Sensitivity Syndrome

3.6.4 Tight Building Syndrome

3.7 Toxic Outbreaks of Historical Significance (e.g., Yusho, Toxic Oil)

\section{Part 4: Therapeutics}

4.1 ABCs - Resuscitation

4.2 Initial Management

4.2.1 Decontamination Strategies

4.2.1.1 Dermal

4.2.1.2 Gastrointestinal
4.2.1.3 Ocular

4.2.2 Enhanced Elimination Techniques

4.2.2.1 Extracorporeal Removal

4.2.2.2 Gastrointestinal Dialysis

4.2.2.3 Urinary Clearance

4.3 Pharmacological Basis of Antidote Use

4.3.1 Antagonize Effects of Poison

4.3.1.1 Enzyme Inhibitors (e.g., Physostigmine)

4.3.1.2 Enzyme Reactivators (e.g., Pralidoxime)

4.3.1.3 Physiological Antagonists (e.g., Calcium, Glucagon)

4.3.1.4 Receptor Antagonists (e.g., Atropine, Flumazenil, Naloxone)

4.3.1.5 Reducing Agent (e.g., Methylene Blue, $\mathrm{N}$-Acetylcysteine)

4.3.2 Dispositional Agents

4.3.2.1 Alcohol Dehydrogenase Inhibitors

4.3.2.2 Antibody/Antivenom

4.3.2.3 Chelators

4.3.2.4 Cyanide Antidotes

4.3.2.5 Enzyme/Cofactor Replacement (e.g., Folinic Acid, Pyridoxine)

4.3.2.6 Oxygen/Hyperbaric Therapy

4.3.3 Other Antidotes

4.4 Supportive and Other Care

4.4.1 Airway Management/Oxygenation/Ventilation

4.4.2 Specific Therapies (e.g., Anticonvulsants, Antidysrhythmics)

4.4.3 Control of Agitation

4.4.4 Control of Blood Pressure and Heart Rate

4.4.5 Control of Temperature

4.4.6 Correct Electrolyte and Acid-base Disturbances

4.4.7 Critical Care Procedures (e.g., Arterial Catheter and Central Line Placement)

4.4.8 Fluid Resuscitation

4.4.9 Patient Monitoring

4.4.10 Adjunctive Therapy (e.g., Granulocyte Colony Stimulating Factor)

4.4.11 Psychiatric Issues

4.4.12 Social Issues

4.4.13 Transplantation

4.5 Withdrawal Syndrome Management

4.5.1 Alcohol

4.5.2 Opioid

4.5.3 Sedative-Hypnotic

4.5.4 Nicotine

4.5.5 Stimulant

4.5.6 Mixed Pattern

4.6 Radiation Exposure Management (e.g., Triage, Medical Therapy) 
4.6.1 Dosimetry (e.g., Exposure Type, Dose Measurement)

4.6.2 Decontamination

4.6.3 Chelation Therapy

\section{Part 5: Assessment and Population Health}

5.1 Criteria for Causal Inference (e.g., Biological Plausibility, Consistency, Dose Response Relationship, Strength of Association, Temporal Relationship)

5.2 Monitoring

5.2.1 Biological Monitoring and Biomarkers (e.g., Population Norms, Indicators of Excessive Exposure)

5.2.2 Environmental Sampling/Exposure Monitoring

5.3 Occupational Assessment and Prevention

5.3.1 Medical Surveillance and Pre-Placement Screening

5.3.2 Exposure Control

5.3.2.1 Engineering Controls

5.3.2.2 Personal Protective Equipment

5.3.2.3 Work Practices and Product Substitution

5.4 Principles of Epidemiology and Study Design

5.4.1 Expert Opinion (e.g., Surveys, Consensus Guidelines)

5.4.2 Poisoning Epidemiology

5.4.3 Study Design

5.4.3.1 Study Types

5.4.3.1.1 Grading of Scientific Evidence

5.4.3.1.2 Non-human Studies (e.g., In Vitro, Animal, etc.)

5.4.3.1.3 Observational Studies

5.4.3.1.3.1 Analytical

5.4.3.1.3.2 Descriptive

5.4.3.1.4 Clinical Trials

5.4.3.2 Validity and Generalizability (e.g., Bias, Confounding, etc.)

5.4.4 Statistical Measures

5.4.4.1 Measures of Association (e.g., Odds Ratios, Relative Risk)

5.4.4.2 Measures of Significance (e.g., $p$-Value, Confidence Interval, etc.)

5.4.4.3 Statistical Power and Sample Size

5.4.4.4 Test Measurements (e.g., Sensitivity, Predictive Values, Likelihood Ratios)

5.5 Risk Assessment and Management

5.5.1 Hazard Identification

5.5.1.1 Review of Prior Studies

5.5.1.1.1 Carcinogenicity Testing

5.5.1.1.2 Human Epidemiological Studies

5.5.2 Dose Response Assessment

5.5.2.1 No Observed and Lowest Observed Adverse Effect Levels (NOAEL, LOAEL)

5.5.2.2 Uncertainty Factors (e.g., Extrapolation-High To Low Dose, Animal To Human, etc.)
5.5.3 Exposure Assessment

5.5.3.1 Exposure Pathways

5.5.3.2 Interpretation of Key Terms (e.g., Recommended Exposure Limit [REL], Permissible Exposure Limit [PEL], Threshold Limit Value [TLV])

5.5.4 Risk Characterization

5.5.5 Target Risks (e.g., 10-5 or 10-6)

5.5.6 Role of Risk Assessment in Formulating Regulations

5.5.7 Risk Perception and Risk Communication

5.6 Poison Centers

5.6.1 Administration/Organization

5.6.2 Data Collection Systems

5.6.3 Surveillance/Interaction with Other Professional Health Organizations

5.7 Response to Hazardous Materials (Hazmat) Incidents, Including Terrorism

5.7.1 Decontamination (Patients and Responders)

5.7.2 Incident Command System, Site Safety and Control Zones

5.7.3 Incident Response Planning and Emergency Preparedness

5.7.4 National Pharmaceutical Stockpile

5.7.5 Regulatory and Legal Background (e.g., HAZWOPER, SARA, CERCLA, RCRA, etc.)

5.8 Role of Federal and International Agencies in Toxicology

5.8.1 Centers for Disease Control and Prevention (CDC)

5.8.1.1 Agency for Toxic Substances and Disease Registry (ATSDR)

5.8.1.2 National Institute for Occupational Safety and Health (NIOSH)

5.8.2 Consumer Product Safety Commission (CPSC)

5.8.3 Drug Enforcement Administration (DEA)

5.8.3.1 Definition and Scheduling of Controlled Substances

5.8.4 Environmental Protection Agency (EPA)

5.8.5 Food and Drug Administration (FDA)

5.8.5.1 Risk Evaluation and Mitigation Strategies

5.8.6 Health Resources and Services Administration (HRSA)

5.8.7 Occupational Safety and Health Administration (OSHA)

5.8.8 World Health Organization (WHO)

5.8.9 Other Agencies

5.9 Injury Prevention

5.9.1 Home Medication Errors

5.9.2 Intentional Poisoning Prevention

5.9.2.1 Depression and Suicidality

5.9.3 Patient Safety/Medical Errors

5.9.3.1 Epidemiology of Medical Errors 
5.9.3.2 General Methodology of Patient Safety

5.9.3.3 Role of Specific Systems Factors in the Commission and Prevention of Medical Errors

5.9.3.4 Use of Process-based and Evidence-based Safety Practices in the Work Environment

5.9.4 Pediatric Exploratory Poisoning Prevention

5.9.4.1 Active Strategies

5.9.4.2 Passive Strategies

5.10 Medical Publishing

5.11 Consultation Resources (e.g., Databases, National Library of Medicine)

Part 6: Analytical and Forensic Toxicology

6.1 Assay Methods and Interpretation

6.1.1 Clinical Drug Testing

6.1.1.1 Confirmatory Tests

6.1.1.1.1 Atomic Absorption

6.1.1.1.2 Gas Chromatography/Mass Spectrometry (GC/MS)

6.1.1.1.3 High-pressure Liquid Chromatography (HPLC)

6.1.1.2 Screening Tests

6.1.1.2.1 Gas Chromatography (GC)

6.1.1.2.2 Immunoassays (e.g., Enzyme-Multiplied Immunoassay Technique [EMIT])

6.1.1.2.3 Colorimetric Tests (e.g., Color Tests, Thin Layer Chromatography)

6.1.1.3 Urine Drug Testing

6.1.1.3.1 Adulteration Techniques

6.1.1.3.2 Analytical Limitations and Interferences

6.1.1.3.3 Interpretative Challenges (e.g., Benzodiazepines, Opioids)

6.1.2 Laboratory Issues

6.1.2.1 Point of Care Testing

6.1.2.2 Quality Assurance/Quality Control (e.g., CLIA)

6.1.3 Routine Toxicology Testing

6.1.4 Special Toxicology Testing

6.1.4.1 Metal Testing

6.1.4.2 Cholinesterase Determinations

6.1.4.3 Hair Analysis (e.g., Metals, Drugs of Abuse)

6.1.5 Therapeutic Drug Monitoring

6.2 Detection and Interpretation of Performance Enhancing Substances
6.3 Laboratory and Other Diagnostic Assessments

6.3.1 Anion Gap

6.3.2 Blood Gases and Co-oximetry

6.3.3 Electrolytes

6.3.4 Hematologic and Coagulation Tests

6.3.5 Liver Function Tests

6.3.6 Osmoles

6.3.7 Urine Analysis (e.g., Urinalysis, Color, Crystals)

6.3.8 Other Supportive Testing

6.3.8.1 Diagnostic Radiologic Imaging

6.3.8.2 Electrocardiography

6.3.8.3 $\mathrm{EMG} / \mathrm{NCV} / \mathrm{EEG}$

6.3.8.4 Pulmonary Function Testing

6.3.8.5 Pulse Oximetry and Related Technology

6.4 Forensics

6.4.1 Chain of Custody

6.4.2 Clandestine Laboratories

6.4.3 Interpretation of Postmortem Drug Levels

6.4.4 Necrokinetics

6.4.5 Selection of Postmortem Specimens

6.4.6 Special Issues

6.4.6.1 Meconium

6.4.6.2 Vitreous Humor

6.5 Legal Drugs of Abuse

6.5.1 Drug-induced Psychomotor Impairment

6.5.2 Performance and Interpretation of Laboratory Testing

6.6 Legal Ethanol

6.6.1 Alcohol and the Law

6.6.2 Alcohol-induced Psychomotor Impairment

6.6.3 Blood and Breath Alcohol Analysis

6.6.4 Collection and Storage of Alcohol Specimens

6.6.5 Forensic Pharmacokinetics of Alcohol

6.6.6 Saliva and Other Media

6.7 Medical Legal Issues

6.7.1 Ethical Standards for Testimony

6.7.2 Expert Witness Standards of Reliability (e.g., Daubert, Frye)

6.7.3 Role of Expert Witness in Various Settings (e.g., Medical Malpractice, Tort)

6.8 Workplace Drug Test Interpretation

6.8.1 Cutoffs

6.8.2 Guidelines for Federal Workplace Drug Testing Programs

6.8.3 Role of Medical Review Officer (MRO) 